\title{
Disease Risk Curves
}

\author{
G. Hughes, F. J. Burnett, and N. D. Havis
}

Crop and Soil Systems Research Group, SRUC, The King's Buildings, West Mains Road, Edinburgh EH9 3JG, UK. Accepted for publication 15 March 2013.

\begin{abstract}
Hughes, G., Burnett, F. J., and Havis, N. D. 2013. Disease risk curves. Phytopathology 103:1108-1114.

Disease risk curves are simple graphical relationships between the probability of need for treatment and evidence related to risk factors. In the context of the present article, our focus is on factors related to the occurrence of disease in crops. Risk is the probability of adverse conse-

modeled as a function of a single factor (specifically the level of disease at an early disease assessment). In both cases, disease risk curves serve as calibration curves that allow the accumulated evidence related to risk to be expressed on a probability scale. When risk is modeled as a function of the level of disease at an early disease assessment, the resulting disease risk curve provides a crop loss assessment model in which the downside is denominated in terms of risk rather than in terms of yield loss.
\end{abstract} quences; specifically in the present context it denotes the chance that disease will reach a threshold level at which crop protection measures can be justified. This article describes disease risk curves that arise when risk is modeled as a function of more than one risk factor, and when risk is
Additional keywords: conditional dependence, disease management, sequential diagnosis.
We know that for a disease to occur in a crop, a susceptible host, the causal pathogen and an environment conducive to disease are necessary. Then, a disease progress curve "represents an integration of all host, pathogen and environmental effects occurring during the epidemic and provides an opportunity to analyze, compare and understand plant disease epidemics" (2). The disease progress curve shows changing disease intensity, measured on an appropriate scale, over a time interval appropriate for the pathosystem in question (e.g., Figure 8.1 of Campbell and Madden [2]). The curve is often sigmoid. In relation to crop protection and disease management, the disease progress curve often provides a basis for crop loss assessment models. For example, a critical point model provides an estimate of yield loss for a given level of disease at a specified time or growth stage (GS); a multiple point model provides an estimate of yield loss for given levels of disease at a number of specified times or GSs; and an area under the disease progress curve (AUDPC) model provides an estimate of yield loss for a given AUDPC between two specified times or GSs (12).

In each case, the estimate of loss can be compared with a prespecified threshold value as a basis for deciding whether or not treatment is required. In using the integrative properties of the disease progress curve as a basis for crop loss assessment, we are in effect assigning to the level of disease at the specified time(s) or GS(s) the properties of an all-encompassing disease risk factor. That is to say, if we want to evaluate disease management practices, or predict the need for their use, the disease progress curve is a basic means to an end (e.g., 13). Indeed, since the pioneering work of van der Plank (30), mathematical models of disease progress, often based on statistical analysis of data obtained from monitoring disease progress over time, have become a standard part of the working epidemiologist's analytical toolkit in relation to crop protection.

More recently, and particularly in the context of forecasting disease, an alternative approach involving the assessment of risk

Corresponding author: G. Hughes; E-mail address: gareth.hughes@ sruc.ac.uk

http://dx.doi.org/10.1094/PHYTO-12-12-0327-R

(c) 2013 The American Phytopathological Society has been in evidence. We use the term "risk" to refer to a probability of adverse consequences (11). In particular, in the present context, the adverse consequences with which we are ultimately concerned relate to crop yield loss resulting from disease. Following Spiegelhalter (23), we avoid use of the term "relative risk". We wish to classify crops in terms of their requirement for protection measures, in order to reduce yield loss to disease. Because the objective is to use the crop protection measures to prevent disease developing to a level that will result in economically significant yield loss, we cannot measure this requirement directly. Instead, we must predict risk-the probability of this requirement-using data relating to one or more important risk factors.

Disease risk curves are simple graphical relationships between the probability of need for treatment and evidence related to risk factors. The purpose of the present article is to describe the application of disease risk curves in the particular context of crop loss assessment. The article is set out as follows. We first discuss the situation in which disease risk is expressed as a function of more than one risk factor. Here, two approaches are outlined, with particular attention paid to the problem of conditional dependence between risk factors. We then discuss the situation in which disease risk is expressed as a function of a single risk factor, specifically the level of disease at an early disease assessment. Finally, a general discussion is provided.

\section{THEORY AND APPROACHES}

Disease risk expressed as a function of more than one risk factor. It makes sense to discuss the situation where disease risk is expressed as a function of more than one risk factor first because in this case examples from the literature can provide a useful context for the present analysis. We utilize two such examples, in both of which a binary logistic regression approach was used as a basis for characterizing the dependence of disease risk on several risk factors, in the context of crop protection decision making.

Example 1. This example is based on the work of Yuen et al. (32) and Twengström et al. (27) on Sclerotinia stem rot of oil seed rape (caused by Sclerotinia sclerotiorum). We can briefly sum- 
marize the analysis of Twengström et al. (27) as follows. Data from a total of 805 untreated crops were analyzed. Retrospectively, crops were divided into two groups: those where spraying would have been economically justified, referred to here as cases (>25\% infected plants, 131 crops) and those where spraying would not have been economically justified, referred to here as controls ( $\leq 25 \%$ infected plants, 674 crops). Stepwise logistic regression procedures were used to identify a set of important risk factors. At this stage we note in passing that the development of a phytopathological diagnostic apparatus typically depends on the deployment of a statistical diagnostic apparatus. In particular, it is desirable to be able to identify risk factors that are independent. On this basis, six important risk factors were identified as follows: number of years since the previous oil seed rape crop, disease incidence in the previous host crop, plant population density, rainfall in the previous 2 weeks, weather forecast, and regional risk for apothecium development (Table 3 of Twengström et al. [27]). Binary logistic regression of crop status (case or control) on explanatory variables (the six important risk factors) was carried out. From this analysis, a risk points score, based on the corresponding regression coefficient, was calculated for each level of each risk factor (Table 4 of Twengström et al. [27]).

Table 4 of Twengström et al. (27) works as follows. The total points score for a crop can be calculated after assessment of all six risk factors. This total is an indicator of the need for treatment. If, in addition, a threshold points score has been identified, we have a predictor ("test" is synonymous) of need for treatment in a crop, such that a total points score above the threshold is a prediction of need for treatment, and a total at or below the threshold is a prediction of no need for treatment. Yuen et al. (32) and Twengström et al. (27) were concerned with the identification of an appropriate threshold points score using receiver operating characteristic (ROC) curve analysis.

In this example, the disease risk curve serves as a calibration curve such that the accumulated risk score can be equated to a probability. To achieve this, we have calculated a binary logistic regression of crop status (case or control) on the single explanatory variable "total points score" for the 805 crops. The required data are available in Twengström et al. (Figure 2 of Twengström et al. [27]). We write the corresponding logistic regression model as follows:

$$
\operatorname{logit}(p \mid X)=\beta_{0}+\beta_{1} X
$$

in which $p$ (probability of need for treatment) depends on a single risk factor $X$ (total risk points score), logit( $\cdot)$ is synonymous with $\ln (\operatorname{odd} s(\cdot))$, and $\beta_{0}$ and $\beta_{1}$ are model parameters. Once we have estimates $\hat{\beta}_{0}$ and $\hat{\beta}_{1}$, we can write $\operatorname{logit}(\hat{p})=\hat{\beta}_{0}+\hat{\beta}_{1} X$, or equivalently:

$$
\hat{p}=\frac{e^{\left(\hat{\beta}_{0}+\hat{\beta}_{1} X\right)}}{1+e^{\left(\hat{\beta}_{0}+\hat{\beta}_{1} X\right)}}=\frac{1}{1+e^{-\left(\hat{\beta}_{0}+\hat{\beta}_{1} X\right)}}
$$

In this case, we obtain $\hat{\beta}_{0}=-6.773$ (standard error $[\mathrm{SE}]=0.512$ ) and $\widehat{\beta}_{1}=0.131(\mathrm{SE}=0.011)$. The corresponding calibration curve is shown in Figure 1.

Based on ROC curve analysis $(27,32)$, Twengström et al. (27) suggested threshold scores of 40 and 50 risk points as a basis for crop spraying recommendations. Using equation 2, the lower threshold (40 risk points) corresponds to a probability of need for treatment of 0.18 and the higher threshold (50 risk points) corresponds to a probability of need for treatment of 0.45 . The ROC curve and the disease risk curve (the calibration curve in Fig. 1) are complementary (e.g., Figure 1 of Lloyd [17]).

Example 2. This example is based on the work of Johnson et al. $(14,15)$ on potato late blight (caused by Phytophthora infestans). We can briefly summarize the analysis of Johnson et al. (14) as follows. Data from a sequence of 28 years (1970 to 1997) were analyzed. Retrospectively, years were divided into cases (outbreak years, 15) and controls (non-outbreak years, 13). Variable selection procedures were used to identify a set of important risk factors. Three important risk factors were identified as follows: outbreak in previous year, number of days with rain in April and May, and number of days with rain in July and August (Table 2 of Johnson et al. [14]). Binary logistic regression of crop status (case or control) was carried out on various combinations of the three important risk factors.

Here, our analysis is based on model 1 of Johnson et al. (14) with two risk factors, each with two levels $(i=1,2)$. We denote "previous year" as risk factor $A$ (outbreak, $A_{1}=1$; non-outbreak, $A_{2}=0$ ) and "number of days with rain in April and May" as risk factor $B$, where for the purpose of our present illustration, we have discretized risk factor $B$ (>12 days, $B_{1}=1 ; \leq 12$ days, $B_{2}=$ $0)$. For the current example, our analysis is based on the data from the Hermiston site (Table 2 of Johnson et al. [14]). We write the corresponding logistic regression model:

$$
\operatorname{logit}(p \mid A, B)=\beta_{0}+\beta_{1} A_{i}+\beta_{2} B_{i}
$$

and then obtain $\hat{\beta}_{0}=-1.853(\mathrm{SE}=0.937), \hat{\beta}_{1}=3.022(\mathrm{SE}=$ $1.023)$, and $\hat{\beta}_{2}=0.973(\mathrm{SE}=1.035)$. Thus, for example, for test outcomes $A_{1}$ and $B_{1}$, we have

$$
\operatorname{logit}\left(\hat{p}_{1,1}\right)=\hat{\beta}_{0}+\widehat{\beta}_{1} A_{1}+\hat{\beta}_{2} B_{1}=2.141
$$

and then

$$
\operatorname{odds}\left(\hat{p}_{1,1}\right)=\exp (2.141)=8.510, \hat{p}_{1,1}=\frac{8.510}{1+8.510}=0.895
$$

Bayes' theorem is a statement of the rule by means of which we can update our initial view, the prior probability of requirement for protection measures, taking into account evidence related to risk factors, to arrive at a posterior probability of requirement for protection measures (31). Particularly when decision-making is based on $>1$ risk factor, more application has been made in clinical disease assessment than in phytopathological disease assess-

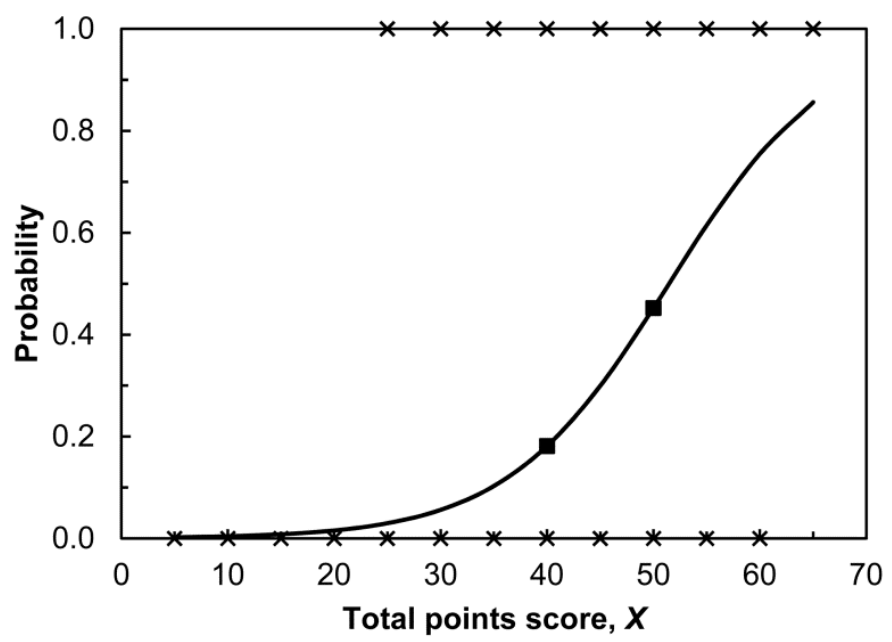

Fig. 1. Sclerotinia stem rot of oil seed rape. Logistic regression of crop status (case or control) on the single explanatory variable "total points score", based on data from Twengström et al. (27). Points $(\times)$ are the binary data for casecontrol status of crops; each point corresponds to more than one crop (Figure 2 of Twengström et al. [27]). The logistic regression curve is $\hat{p}=1 /\left(1+e^{-\left(\hat{\beta}_{0}+\hat{\beta}_{1} x\right)}\right), \quad \hat{\beta}_{0}=-6.773, \hat{\beta}_{1}=0.131 ;$ the points $(\mathbf{\square})$ indicate the threshold risk scores chosen by Twengström et al. (27) on the basis of receiver operating characteristic curve analysis. 
ment. Here, after a generic introduction, we will provide an analysis via Bayes' theorem of the Hermiston potato late blight data for comparison with the logistic regression approach.

The true status of a crop is denoted $D_{j}(j=1,2)$. Here, $D_{1}$ denotes that the true status of a crop is that of disease outbreak, or need for treatment, and $D_{2}$ denotes that the true status is that of no outbreak, or no need for treatment. The prior (i.e., pre-test) probability of a crop's requirement for treatment is denoted $\operatorname{Pr}\left(D_{1}\right)$. Here, this is a point probability based in some appropriate way on our previous experience. Note, however, that for brevity, we omit this element of conditionality from our notation for the prior. Since we have a simple hypothesis (a crop is either $D_{1}$ or $D_{2}$ ), we have $\operatorname{Pr}\left(D_{2}\right)=1-\operatorname{Pr}\left(D_{1}\right)$. As with logistic regression, some calculations are simplified if we work in terms of odds rather than probabilities, and further simplification may result if we work in terms of log-odds. For numerical calculations we will use natural (base $e$ ) logarithms (written as ln), other logarithms are specified by writing the base as a subscript (e.g., common logarithms are written as $\log _{10}$ ); if the base is unspecified (as in log-odds, above), the reference is generic.

Here, for simplicity, we deal with the case of risk factors that provide a binary test outcome. Thus, the outcome of a test is denoted generically $T_{i}(i=1,2) . T_{1}$ denotes that the predicted status of a crop is that of disease outbreak, or need for treatment, and $T_{2}$ denotes that the predicted status is that of no outbreak, or no need for treatment. We can now write the posterior (i.e., post-test) probabilities as $\operatorname{Pr}\left(D_{j} \mid T_{i}\right)$ and the corresponding odds as $\operatorname{odds}\left(D_{j} \mid T_{i}\right)$. We denote the likelihood ratios as

$$
L R_{i}=\frac{\operatorname{Pr}\left(T_{i} \mid D_{1}\right)}{\operatorname{Pr}\left(T_{i} \mid D_{2}\right)}
$$

$(i=1,2) . L R_{1}$ tells us how much more likely is a $T_{1}$ test outcome from $D_{1}$ subjects as compared with $D_{2}$ subjects. $L R_{2}$ tells us how much less likely is a $T_{2}$ test outcome from $D_{1}$ subjects as compared with $D_{2}$ subjects.

Now, we can write out Bayes' theorem in equation format (in the simple case under discussion, the Bayes factors are likelihood ratios) $(7,16)$. There are several versions; here we adopt the logodds format (referred to by Tribus [26] as the evidence form of Bayes' equation). For a binary test with a $D_{1}$ subject, the test outcome may be $T_{1}$, in which case:

$$
\log \left(\operatorname{odds}\left(D_{1} \mid T_{1}\right)\right)=\log \left(\text { odds }\left(D_{1}\right)\right)+\log \left(L R_{1}\right)
$$

or the test outcome may be $T_{2}$, in which case:

$$
\log \left(\operatorname{odds}\left(D_{1} \mid T_{2}\right)\right)=\log \left(\operatorname{odds}\left(D_{1}\right)\right)+\log \left(L R_{2}\right)
$$

Similarly, for a $D_{2}$ subject, the test outcome may be $T_{1}$, in which case:

$$
\log \left(\operatorname{odds}\left(D_{2} \mid T_{1}\right)\right)=\log \left(\operatorname{odd} s\left(D_{2}\right)\right)-\log \left(L R_{1}\right)
$$

or the test outcome may be $T_{2}$, in which case:

$$
\log \left(\operatorname{odd} s\left(D_{2} \mid T_{2}\right)\right)=\log \left(\operatorname{odd} s\left(D_{2}\right)\right)-\log \left(L R_{2}\right)
$$

Note that a binary test will be calibrated so that $\log \left(L R_{1}\right)>0$ and $\log \left(L R_{2}\right)<0$, in which case a $T_{1}$ test outcome for a $D_{1}$ subject (a "true positive") results in $\log \left(\right.$ odd $\left.s\left(D_{1} \mid T_{1}\right)\right)>\log \left(\right.$ odd $\left.s\left(D_{1}\right)\right)$ (equation 4), a $T_{2}$ test outcome for a $D_{1}$ subject (a "false negative") results in $\log \left(\operatorname{odd} s\left(D_{1} \mid T_{2}\right)\right)<\log \left(\operatorname{odd} s\left(D_{1}\right)\right)$ (equation 5), a $T_{1}$ test outcome for a $D_{2}$ subject (a "false positive") results in $\log \left(\operatorname{odds}\left(D_{2} \mid T_{1}\right)\right)<\log \left(\right.$ odds $\left.\left(D_{2}\right)\right)$ (equation 6), and a $T_{2}$ test outcome for a $D_{2}$ subject (a "true negative") results in $\log \left(\operatorname{odd} s\left(D_{2} \mid T_{2}\right)\right)>\log \left(\right.$ odds $\left.\left(D_{2}\right)\right)$ (equation 7). In the extensive writings of Good on the subject (e.g., 6-8), the log-likelihood ratio is referred to as the "weight of evidence".

Tribus (26) provides an example of Bayes' theorem applied in a diagnostic scenario, based on an industrial quality control problem in which a lot is sampled (such that the sample size is small relative to the size of the lot) as a basis for deciding whether the proportion defective items is unacceptable $\left(D_{1}\right.$ in the current notation) or acceptable ( $D_{2}$ in the current notation). This is achieved by sequentially testing items from the lot without replacement, and noting how the posterior log-odds for hypothesis $D_{1}$ and hypothesis $D_{2}$ change as the testing progresses. In this situation, the sequential tests can be regarded as independent. Bayes' theorem provides a basis for this calculation. Suppose the test outcomes are $T_{1}, T_{1}, T_{2}, T_{1}, T_{1}$ (in this example, the same test is carried out on a different item in each instance). The posterior $\log$-odds for hypothesis $D_{1}$ changes as follows:

First test $\quad \log \left(\right.$ odds $\left.\left(D_{1} \mid T_{1}\right)\right)=\log \left(\operatorname{odd} s\left(D_{1}\right)\right)+\log \left(L R_{1}\right)$

Second test $\quad \log \left(\operatorname{odds}\left(D_{1} \mid T_{1}, T_{1}\right)\right)=\log \left(\operatorname{odds}\left(D_{1} \mid T_{1}\right)\right)+\log \left(L R_{1}\right)$

Third test $\quad \log \left(\operatorname{odd} s\left(D_{1} \mid T_{1}, T_{1}, T_{2}\right)\right)=$ $\log \left(\operatorname{odd} s\left(D_{1} \mid T_{1}, T_{1}\right)\right)+\log \left(L R_{2}\right)$

Fourth test $\log \left(\operatorname{odds}\left(D_{1} \mid T_{1}, T_{1}, T_{2}, T_{1}\right)\right)=$ $\log \left(\operatorname{odds}\left(D_{1} \mid T_{1}, T_{1}, T_{2}\right)\right)+\log \left(L R_{1}\right)$

Fifth test

$$
\begin{aligned}
& \log \left(\operatorname{odds}\left(D_{1} \mid T_{1}, T_{1}, T_{2}, T_{1}, T_{1}\right)\right)= \\
& \quad \log \left(\operatorname{odds}\left(D_{1} \mid T_{1}, T_{1}, T_{2}, T_{1}\right)\right)+\log \left(L R_{1}\right)
\end{aligned}
$$

Tribus illustrates this (Figure III-3 of Tribus [26]) on a graph that shows both a (linear) posterior $\log _{10} 0.1$-odds scale and the corresponding posterior probability on its two vertical axes and the sequence of tests on the horizontal axis. The calibration of the vertical axes is such that if $\log _{10^{0.1}}($ odds $($ outcome $))=y$, the corresponding probability is

$$
\operatorname{Pr}(\text { outcome })=\frac{1}{1+10^{-0.1 y}}
$$

The basic property of the analysis described above is that (at each stage) final log-odds equals initial log-odds plus the loglikelihood ratio for the test outcome.

Glass (5) provides an overview of the application of this diagnostic methodology in a clinical context. Further discussion and examples are provided by, for example, Rembold and Watson (21) and Van den Ende et al. $(28,29)$. One difficulty that arises here is that the assessment of risk for an individual subject does not allow a sampling procedure, such that the sequential tests may be regarded as independent, as in Tribus' (26) example. Nor is there any built-in statistical diagnostic procedure (such as there is with logistic regression) to test for conditional dependence among a set of risk factors at the variable selection stage. Either an assumption of independence among risk factors included in the diagnostic work-up is adopted with some caution; or the weights of evidence provided by the log-likelihood ratios must be adjusted to take account of their possible conditional dependence. Here, one example of the latter approach, as discussed by Spiegelhalter and Knill-Jones (24) and Spiegelhalter (22), is applied to the potato late blight data of the current example.

First, based on Table 2 by Johnson et al. (14) (and recalling our discretization of the days with rain data), we take the prior prob- 
ability of an outbreak year as $\operatorname{Pr}\left(D_{1}\right)=15 / 28=0.536$, and calculate the likelihood ratios for the Hermiston potato late blight data as follows:

$$
\begin{aligned}
& \operatorname{LR}(A)_{1}=\frac{12}{15} / \frac{2}{13}=5.200 ; \quad \operatorname{LR}(A)_{2}=\frac{3}{15} / \frac{11}{13}=0.236 \\
& L R(B)_{1}=\frac{11}{15} / \frac{6}{13}=1.589 ; \quad \operatorname{LR}(B)_{2}=\frac{4}{15} / \frac{7}{13}=0.495
\end{aligned}
$$

Then (for the example case of test outcomes $A_{1}$ and $B_{1}$, as above) we have

$$
\ln \left(\text { odds }\left(D_{1} \mid A_{1}, B_{1}\right)\right)=\ln \left(\text { odds }\left(D_{1}\right)\right)+\ln \left(L R(A)_{1}\right)+\ln \left(L R(B)_{1}\right)=2.255
$$

Noting that $2.255>2.141$, we observe that the increase in ln-odds in relation to the evidence-compared with the previously obtained result based on the equivalent logistic regression-is (slightly) overestimated. The difference is slight because we are reanalyzing an example where the two explanatory variables in the model had been chosen following previous use of a variable selection procedure with the aim of finding risk factors that were independent. Thus, the small difference observed here corroborates the variable selection procedures adopted by Johnson et al. (14).

Notwithstanding, we can still demonstrate the approach discussed by Spiegelhalter and Knill-Jones (24) and Spiegelhalter (22) to the calculation of adjusted weights of evidence. Thus, we calculate a logistic regression (equation 3 ) but instead of using 1 for a positive test and 0 for a negative test, we use the ln-likelihood ratios (i.e., the raw weights of evidence) for the test outcomes, as follows. We denote "previous year" as risk factor $A$ (outbreak, $A_{1}=\ln \left(L R(A)_{1}\right)$; non-outbreak, $A_{2}=\ln \left(L R(A)_{2}\right)$ ) and "number of days with rain in April and May" as risk factor $B$ (discretized as previously: $>12$ days, $B_{1}=\ln \left(\operatorname{LR}(B)_{1}\right)$; $\leq 12$ days, $\left.B_{2}=\ln \left(L R(B)_{2}\right)\right)$. A continuity correction may be applied to the calculation of the weights of evidence $(22,24)$ but we have not done so here. For the current example, our analysis is based on the data from the Hermiston site (Table 2 of Johnson et al. [14]). We now obtain $\widehat{\beta}_{0}=0.143(\mathrm{SE}=0.506), \widehat{\beta}_{1}=0.978(\mathrm{SE}=0.331)$, and $\hat{\beta}_{2}=0.834(\mathrm{SE}=0.888)$. Now, for test outcomes $A_{1}$ and $B_{1}$, we have

$$
\operatorname{logit}\left(\hat{p}_{1,1}\right)=\widehat{\beta}_{0}+\widehat{\beta}_{1} \ln \left(L R(A)_{1}\right)+\widehat{\beta}_{2} \ln \left(L R(B)_{1}\right)=2.141
$$

This provides a constant term $\hat{\beta}_{0}$ interpretable as the $\ln$ (prior odds), and $\hat{\beta}_{1} \ln \left(L R(A)_{1}\right)$ and $\hat{\beta}_{2} \ln \left(L R(B)_{1}\right)$ interpretable as the adjusted weights of evidence provided by observations $A_{1}$ and $B_{1}$, respectively. Thus, we have emulated the result of the previous logistic regression analysis of the same data via Bayes' theorem. To do so, we have calculated shrinkage estimates to take account of conditional dependence between explanatory variables (3). We note that the values $\hat{\beta}_{1} \approx 1$ and $\hat{\beta}_{2} \approx 1$ obtained here are further corroboration of the variable selection procedures of Johnson et al. (14) (under independence, $\widehat{\beta}_{1}=\widehat{\beta}_{2}=1$ ).

The disease risk curve is again a calibration curve. If $\ln ($ odds $($ outcome $))=y$, the corresponding probability is $\operatorname{Pr}($ outcome $)=1 /\left(1+e^{-y}\right)$. Because no data are required to draw this graph, we have not provided a figure here (if interested, see Figure 2 of Rembold and Watson [21]). The graph of $\operatorname{Pr}$ (outcome) against $y$ is monotone increasing; therefore, an optimistically large value of $y$ (and the corresponding $\operatorname{Pr}$ (outcome) value) provided by sequential application of Bayes' theorem for a conditionally dependent set of risk factors would be adjusted appropriately downward by application of Spiegelhalter and Knill-Jones' (24) analysis.

Overview of examples 1 and 2. Our analysis of examples 1 and 2 illustrates two different approaches to the problem of conditional dependence between risk factors. The multiple logistic regression approach takes account of conditional dependence in the process of parameter estimation but does not allow for sequential diagnosis. The sequential Bayes approach allows for sequential diagnosis but does not, in its naïve application, take account of conditional dependence between risk factors. We show how the sequential Bayes approach can be adjusted to take account of conditional dependence. When all the risk factors are binary variables and there are no missing values in the data, the adjusted sequential Bayes approach is identical to the logistic regression approach, with the additional feature that sequential diagnosis is facilitated. We return to this in the Discussion. In both example 1 and example 2, the disease risk curve serves as a calibration curve, allowing expression of disease risk on a probability scale.

Disease risk expressed as a function of an early disease assessment. We now consider disease risk curves based on binary logistic regression with a single explanatory variable, where the explanatory variable in question is a disease assessment. We note that this approach differs from most applications of binary logistic regression in crop protection, where the explanatory variables typically include environmental- and host-related factors but not a disease assessment on the crop in question. Among the few relevant examples from the literature, Fabre et al. (Figure 2 in Fabre et al. [4]) show an example where the single explanatory variable is a population density assessment for an aphid vector species, which we might consider an indirect disease assessment. Makowski et al. (18) implicitly discuss an example where the single explanatory variable is an assessment of percentage of contaminated flowers but do not provide details of the regression analysis. Pethybridge et al. (Figure 5B in Pethybridge et al. [20]) provide a disease risk curve by averaging environmental risk factors over the period of the study and then calculating a logistic regression with percent infested seed as the single explanatory variable. In these examples and the two that we present here, the explanatory variable is a disease assessment of some kind, but rather than yield (or yield loss), it is the probability of need for treatment that is the response variable. Still, the fact that our logistic regression-based models use an explanatory variable based on disease assessment emphasizes the fact that these disease risk curves are indeed crop loss assessment models but of a type not considered by, for example, James (12) or Teng and Gaunt (25).

Example 3. Here, we present a subset of data from a large-scale study of Ramularia leaf spot (RLS, caused by Ramularia collocygni) $(9,10)$. From a total of 306 barley crops (including both spring- and winter-sown crops) untreated for RLS, each was retrospectively classified as either a case (treatment would have been economically justified, 207 crops) or a control (treatment would not have been economically justified, 99 crops) based on the recorded yield loss. For 37 of these crops (22 cases and 15 controls), AUDPC had been calculated from RLS assessments made during the growing season.

In the analysis presented here, the probability of need for treatment, $p$, depends on a single risk factor, $X$ (AUDPC). The corresponding linear logistic model is equation 1 and, following parameter estimation, we obtain equation 2 , in this case with $\widehat{\beta}_{0}=-1.864$ $(\mathrm{SE}=0.865)$ and $\hat{\beta}_{1}=0.0137(\mathrm{SE}=0.0052)$ (Fig. 2). From Figure 2 we see that, at high values of AUDPC, risk (i.e., probability of need for treatment) is high, as would be expected. At the lower end of the AUDPC scale, we calculate the following values: $\hat{p}=$ 0.14 when $\mathrm{AUDPC}=0$ and $\hat{p}=0.30$ when $\mathrm{AUDPC}=75$ (the economic threshold value for malting-quality barley crops based on 2010 prices) (10). 
In Figure 2, our disease risk curve is characterized by a twoparameter model. The parameter $\beta_{0}$ characterizes risk when AUDPC $=0$. We can think of this as risk attributable to risk factors (e.g., high RLS in previous crop, choice of a susceptible variety, early sowing date) preceding the observation of disease symptoms in the crop. This is a conditional risk that is only realized if the pathogen is present and disease eventually results. The parameter $\beta_{1}$ characterizes how risk increases as AUDPC increases. We can think of this as risk attributable to factors that increase the rate of disease progress in the crop (e.g., prolonged periods of leaf surface wetness after symptoms have been observed). We have a simple way to apportion risk between these two sources, because we can calculate the risk when AUDPC $=0$ and the risk when AUDPC is at the relevant threshold value. For example, on the basis of Figure 2, at the economic threshold for treatment for malting-quality barley crops, approximately half the risk is attributable to factors that can be assessed preceding disease assessment in the current crop.

Example 4. It is not always the case that a threshold value for categorization of cases and controls is known. In such cases, more than one such threshold may be investigated (e.g., 19). The example here is based on a study of eyespot (caused by Oculimacula acuformis and $O$. yallundae) development and yield losses in winter wheat (1). We present a data set comprising 299 winter wheat crops, untreated for eyespot disease, for which percent eyespot disease assessments were made at both GS31-32 (recorded as eyespot incidence) and GS70-80 (recorded as eyespot index) (1). There is a clear association between percent disease as measured at the early and late assessments (Fig. 3).

In regular agronomic practice, treatment is carried out in order to prevent the GS70-80 (end-of-season) percent disease reaching a prespecified threshold level. Among untreated experimental crops, those above this level are retrospectively classified as cases (treatment was required); and those at or below it are retrospectively classified as controls (treatment was not required). Here, rather than select a single threshold value for analysis, five different calculations were carried out, each with a different threshold used to classify crops as either cases or controls on the basis of an eyespot disease assessment at GS70-80 (recorded as percent eyespot index): 10, 15, 20, 30, and 45\% (Fig. 3). In the analysis presented here, the probability of need for treatment $p$ depends on a single risk factor "percent eyespot incidence at GS31-32", denoted $X$. The corresponding linear logistic model is equation 1 , and following parameter estimation, we obtain a version of equa-

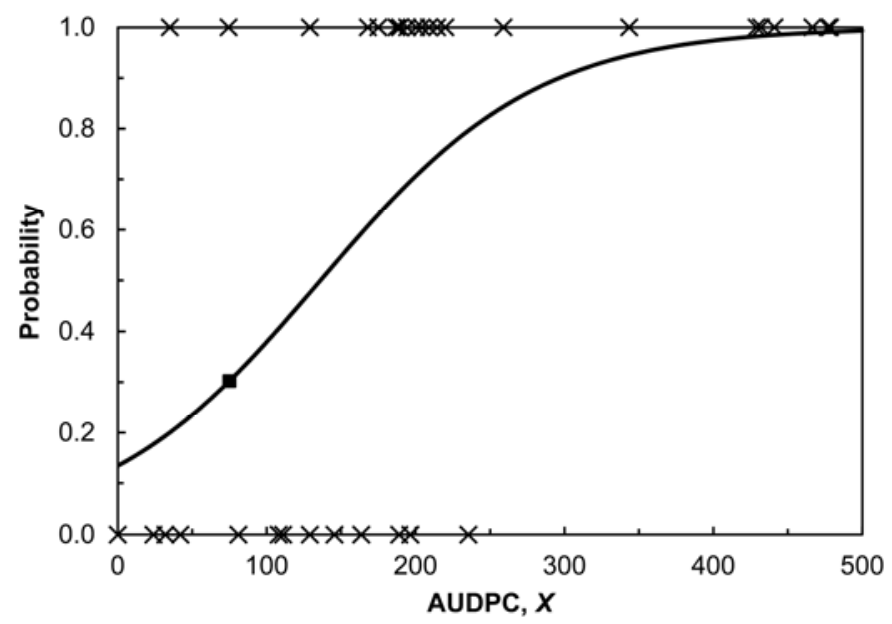

Fig. 2. Ramularia leaf spot of barley. Logistic regression of crop status (case or control) on the single explanatory variable "area under the disease progress curve" (AUDPC). Data points are indicated $(x)$. The logistic regression curve is $\hat{p}=1 /\left(1+e^{-\left(\hat{\beta}_{0}+\hat{\beta}_{1} X\right)}\right), \hat{\beta}_{0}=-1.864, \hat{\beta}_{1}=0.0137$; the point $(\square)$ indicates the economic threshold value for malting-quality barley crops based on 2010 prices. tion 2 for each threshold, with corresponding parameter estimates, as given in Table 1 .

In Figure 4 we have a set of disease risk curves, each characterized by a two-parameter model (Table 1). The parameter $\beta_{0}$ characterizes risk when percent eyespot incidence at GS31-32 = 0 . This is the conditional risk attributable to risk factors (e.g., region, soil type, previous crop, tillage, and early sowing date) preceding the observation of disease symptoms in the crop. The parameter $\beta_{1}$ characterizes how risk increases as percent eyespot incidence at GS31-32 increases. When percent eyespot incidence at GS31-32 is low, probability of need for treatment depends mainly on the choice of case-control threshold. Risk-sensitive decision-makers would tend to adopt relatively low thresholds (e.g., 10 to $15 \%$ ) so that more crops will be classified as cases; risktolerant decision-makers would tend to adopt relatively high thresholds (e.g., 30 to $45 \%$ ) so that fewer crops will be classified as cases. Probability of need for treatment increases with increasing percent eyespot incidence at GS31-32. At very high levels of percent eyespot incidence at GS31-32, we can see that the choice of case-control threshold has little influence on probability of need for treatment. Even if a risk-tolerant decisionmaker initially was of the opinion that eyespot risk was low (expressed as a high case-control threshold), the evidence provided by a very high level of percent eyespot incidence at GS31-32 eventually outweighs that initial view. From this analysis, the more sensitive a decision-maker is to eyespot risk, the more important is $\beta_{0}$ as a component of the total risk. For a decisionmaker tolerant of eyespot risk, $\beta_{0}$ is less important as a component of the total risk, and crop protection decision making is based more on the outcome of the disease assessment at GS31-32.

Overview of examples 3 and 4. Our analysis of examples 3 and 4 illustrates disease risk curves that are simple graphical relationships between disease risk and the level of disease determined at an early assessment. Two features are notable. First, the parameterization of these disease risk curves allows a two-stage risk accumulation, the first stage corresponding to conditional risk attributable to risk factors that precede the disease assessment and the second stage corresponding to risk attributable to the level of

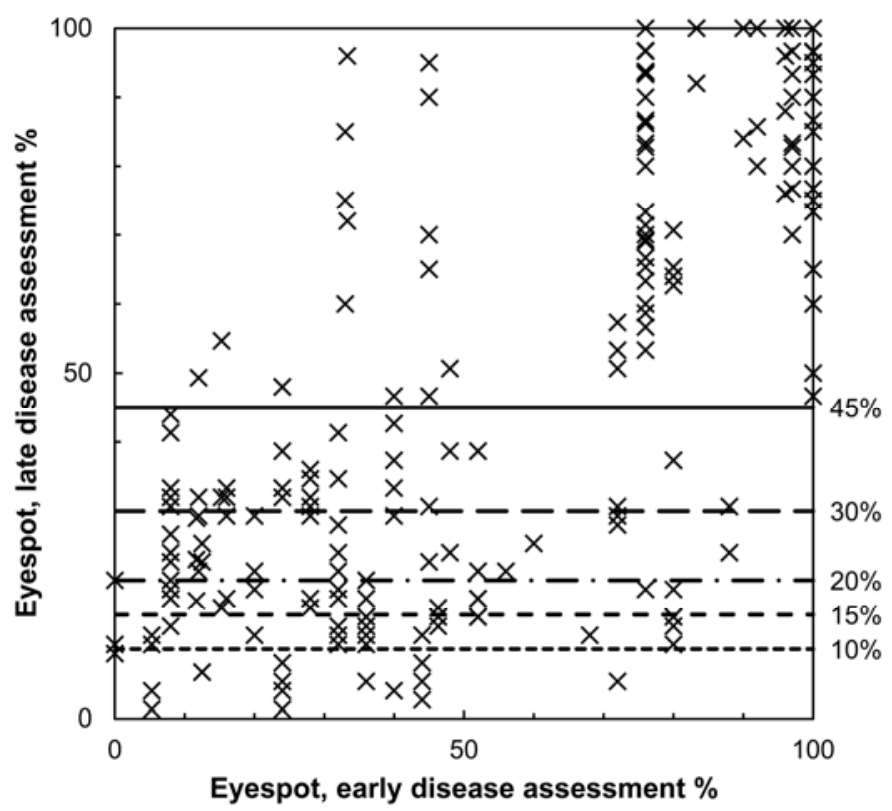

Fig. 3. Eyespot disease of winter wheat. Association between the late disease assessment (eyespot index, growth stage [GS]70-80) and the early disease assessment (eyespot incidence, GS31-32) for 299 untreated crops. Horizontal lines represent different choices of threshold eyespot index, above which crops were classified as cases, at or below which they were classified as controls: $10 \%$ (282 cases, 17 controls); $15 \%$ (255 cases, 44 controls); $20 \%$ (232 cases, 67 controls); $30 \%$ (201 cases, 98 controls); and 45\% (173 cases, 126 controls). 
disease at the disease assessment. Second, the choice of threshold for classification of crops as cases and controls allows the attitude to risk of a decision maker to be taken into account in formulation of the disease risk curve. We return to these features in the Discussion. The disease risk curves again serve as calibration curves that allow the expression of disease risk on a probability scale.

\section{DISCUSSION}

Disease risk curves arise in the context of crop protection decision making, when we model the probability of need for treatment in relation to risk factors. Disease risk curves are simple graphical relationships between risk (i.e., probability of need for treatment) and evidence related to risk factors. Such evidence may be characterized in different ways in different pathosystems; however, a disease risk curve serves as a calibration curve by relating the amount of evidence accumulated to a probability scale. Here, we have described the disease risk curves that arise when risk is expressed as a function of more than one risk factor (the multivariate case) and when risk is expressed as function of a single risk factor, the level of disease at an early assessment (the univariate case).

In the multivariate case, simultaneous estimation of model parameters in a multiple logistic regression takes account of conditional dependence between risk factors. If a risk points scale is adopted to provide a common currency for risk accumulation, the disease risk curve provides a calibration between risk points and probability of need for treatment. However, sequential accumulation of risk over the growing season is not accommodated by models based on simultaneous estimation of model parameters. In a clinical context, sequential accumulation of risk over the process of a diagnostic work-up has sometimes been accomplished by calculation of log-likelihood ratios (weights of evidence) associated with levels of important risk factors, followed by serial application of Bayes' theorem. The problem here is that risk may be overestimated because conditional dependence between risk factors is not taken into account. Van den Ende et al. (28) acknowledge this difficulty in connection with their graphical methods of risk calculation and display.

One way round this problem is to estimate shrinkage parameters that provide for calculating adjusted weights of evidence. Spiegelhalter and Knill-Jones (24) describe one such analysis, which "will allow sequential calculation of accumulating scores." On this basis, the kind of graphical plot discussed by Van den Ende et al. $(28,29)$ could be constructed using adjusted weights of evidence rather than the raw values. This is an attractive propo-

TABLE 1. Eyespot disease of winter wheat ${ }^{\mathrm{a}}$

\begin{tabular}{lcrcc}
\hline Threshold & $\begin{array}{c}\text { Regression } \\
\text { term }\end{array}$ & Estimate & Standard error & $P$ value \\
\hline $10 \%$ & $\beta_{0}$ & 1.1080 & 0.3888 & 0.004 \\
& $\beta_{1}$ & 0.0391 & 0.0103 & $<0.001$ \\
$15 \%$ & $\beta_{0}$ & -0.0287 & 0.2865 & $0.920^{\mathrm{b}}$ \\
& $\beta_{1}$ & 0.0372 & 0.0063 & $<0.001$ \\
$20 \%$ & $\beta_{0}$ & -0.9405 & 0.2791 & $<0.001$ \\
& $\beta_{1}$ & 0.0439 & 0.0058 & $<0.001$ \\
$30 \%$ & $\beta_{0}$ & -2.1980 & 0.3206 & $<0.001$ \\
& $\beta_{1}$ & 0.0542 & 0.0059 & $<0.001$ \\
$45 \%$ & $\beta_{0}$ & -4.4470 & 0.5100 & $<0.001$ \\
& $\beta_{1}$ & 0.0799 & 0.0080 & $<0.001$ \\
\hline
\end{tabular}

${ }^{a}$ Logistic regression of crop status (case or control) on the single explanatory variable "percent eyespot incidence at GS31-32", with five different casecontrol thresholds. Data are shown in Figure 3.

b The $P$ value for this estimate of $\beta_{0}$ is strikingly different from the others in the table simply because, in this case, the estimate is so close to zero. Numerically, values of both the estimate itself and its standard error are consistent with the rest of the table. sition. In crop protection decision making, there is a natural chronology to the accumulation of risk, starting with factors relating to the site (e.g., soil type, previous crop), through agronomic factors relating to the crop in question (e.g., cultivar selection, sowing date), to environmental factors relating to the crop and its interaction with the pathogen in question (e.g., rainfall, temperature). Used with adjusted weights of evidence, the kind of graphical plot discussed by Van den Ende et al. (Figure 2 in Van den Ende et al. [28]; Figure in Van den Ende et al. [29]) provides a basis for a sequential disease risk curve that portrays this chronology.

In the univariate case, we have a two-parameter model in which probability of need for treatment depends on a single explanatory variable, data for which are provided by a disease assessment of some form. Essentially, we have a crop loss assessment model in which the downside is denominated in terms of risk rather than in terms of yield loss. Risk accumulation is a two-stage process. When the intercept on the vertical (probability) axis of the disease risk curve is $>0$, a crop is exposed to risk consequent on decisions made before the disease assessment is made. The realization of this risk is conditional on subsequent disease occurrence in the crop. Thus, the second stage of risk accumulation is the disease assessment itself.

We note that the conditional (pre-disease assessment) component of risk relates to risk factors that have been identified (e.g., from previous epidemiological studies) but need not be observed in the process of risk assessment. In fact, the level of this conditional component of risk depends on how the threshold between cases (crops that would benefit from treatment) and controls (crops in which treatment is unnecessary) is defined. This allows versions of the crop loss assessment model to be calibrated for different attitudes to risk among decision makers. For example, in the case of eyespot disease of winter wheat (example 4), each different case-control threshold provides a different intercept on the vertical (probability) axis of the disease risk curve (Fig. 4). A risk-sensitive decision maker (low case-control threshold) will already have accumulated a lot of (conditional) risk before undertaking a disease assessment. A risk-tolerant decision-maker (high

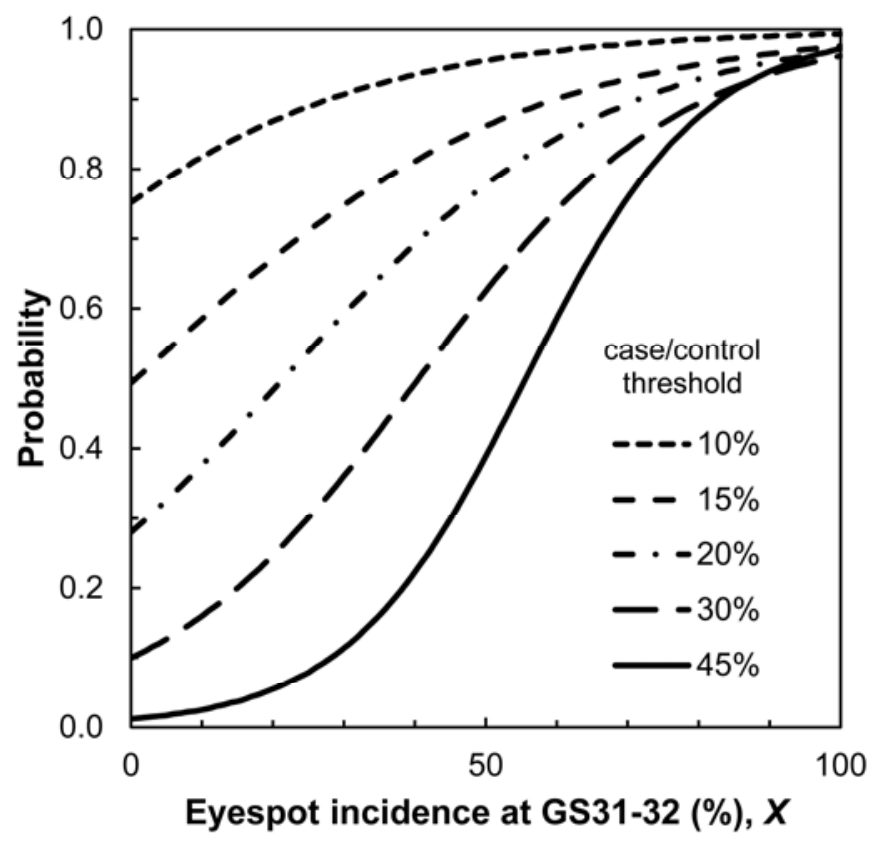

Fig. 4. Eyespot disease of winter wheat. Logistic regression of crop status (case or control) on the single explanatory variable "percent eyespot incidence at growth stage [GS]31-32". Data are shown in Figure 3 and the parameter estimates for the logistic regression curves $\hat{p}=1 /\left(1+e^{-}\left(\hat{\beta}_{0}+\hat{\beta}_{1} X\right)\right)$ are shown in Table 1. 
case-control threshold) accumulates less (conditional) risk before undertaking a disease assessment. In this case, the risk depends mainly on the outcome of the disease assessment.

Finally, returning to where we started, with disease progress curves, most epidemiologists will be familiar with the simple twoparameter models introduced by van der Plank (30), in which disease at any time depends on initial inoculum and average infection rate. Disease control can then be thought of in terms of reducing either or both of these. We can do the same with our disease risk curve based on a simple two-parameter binary logistic regression model. Risk accumulation may depend both on the levels of factors established before the onset of disease and on the level of disease at a subsequent disease assessment. Disease control can then be thought of in terms of reducing either or both of these components of risk.

\section{ACKNOWLEDGMENTS}

SRUC receives grant-in-aid from the Scottish Government. The work on which example 3 is based was funded by HGCA (HCGA is the cereals and oilseeds division of the Agriculture and Horticulture Development Board, AHDB) and the Scottish Government. The work on which example 4 is based was funded by HGCA, with in-kind contributions from British Wheat Breeders, Bayer CropScience, Syngenta Crop Protection Ltd., BASF, and Harper Adams University College. We also thank our research partners NIAB TAG (The National Institute of Agricultural Botany, The Arable Group Limited) and the University of Nottingham for field trial data, and all the farmers who assisted with the provision of field trial sites.

\section{LITERATURE CITED}

1. Burnett, F., Butler-Ellis, C., Hughes, G., Knight, S., and Ray, R. 2012. Forecasting Eyespot Development and Yield Losses in Winter Wheat. Project Report Number 491. HGCA, Kenilworth, UK

2. Campbell, C. L., and Madden, L. V. 1990. Introduction to Plant Disease Epidemiology. John Wiley \& Sons, Inc., New York.

3. Copas, J. B. 1993. The shrinkage of point scoring methods. J. R. Stat. Soc. C 42:315-331.

4. Fabre, F., Plantegenest, M., and Yuen, J. 2007. Financial benefit of using crop protection decision rules over systematic spraying strategies. Phytopathology 97:1484-1490.

5. Glass, R. D. 1996. Diagnosis, a Brief Introduction. Oxford University Press, Melbourne, Australia.

6. Good, I. J. 1979. Studies in the history of probability and statistics. XXXVII. A. M. Turing's statistical work in World War II. Biometrika 66:393-396.

7. Good, I. J. 1985. Weight of evidence: A brief survey. In: Bayesian Statistics 2. J. M. Bernardo, M. H. DeGroot, D. V. Lindley, and A. F. M. Smith, eds. Elsevier Science Publishers B.V. (North-Holland), Amsterdam, The Netherlands.

8. Good, I. J. 2003. The accumulation of imprecise weights of evidence. SIPTA Newsl. 1:2-8.

9. Havis, N. D., Oxley, S. J. P., and Burnett, F. J. 2012. Advances in control of Ramularia collo-cygni. Pages 119-124 in: Proc. Crop Prot. North. Britain, Dundee, UK.

10. Havis, N. D., Oxley, S. J. P., Burnett, F. J., and Hughes, G. 2012. Epi- demiology of Ramularia collo-cygni. Pages 125-130 in: Proc. Crop Prot. North. Britain, Dundee, UK.

11. Hood, C., Rothstein, H., and Baldwin, R. 2004. The Government of Risk-Understanding Risk Regulation Regimes. Oxford University Press, Oxford.

12. James, W. C. 1974. Assessment of plant diseases and losses. Annu. Rev. Phytopathol. 12:27-48.

13. Jeger, M. 2004. Analysis of disease progress curves as a basis for evaluating disease management practices. Annu. Rev. Phytopathol. 42:6182.

14. Johnson, D. A., Alldredge, J. R., and Hamm, P. B. 1998. Expansion of potato late blight forecasting models for the Columbia Basin of Washington and Oregon. Plant Dis. 82:642-645.

15. Johnson, D. A., Alldredge, J. R., and Vakoch, D. L. 1996. Potato late blight forecasting models for the semiarid environment of south-central Washington. Phytopathology 86:480-484.

16. Kass, R. E., and Raftery, A. E. 1995. Bayes factors. J. Am. Stat. Assoc. 90:773-795.

17. Lloyd, C. J. 2002. Estimation of a convex ROC curve. Stat. Probabil. Lett. 59:99-111.

18. Makowski, D., Taverne, M., Bolomier, J., and Ducarne, M. 2005. Comparison of risk indicators for Sclerotinia control in oilseed rape. Crop Prot. 24:527-531.

19. Nita, M., Ellis, M. A., and Madden, L. V. 2008. Variation in disease incidence of Phomopsis cane and leaf spot of grape in commercial vineyards in Ohio. Plant Dis. 92:1053-1061.

20. Pethybridge, S. J., Gent, D. H., and Hay, F. S. 2011. Epidemics of ray blight on pyrethrum are linked to seed contamination and overwintering inoculum of Phoma ligulicola var. inoxydabilis. Phytopathology 101:1112-1121.

21. Rembold, C. M., and Watson, D. 1988. Posttest probability calculation by weights, a simple form of Bayes' theorem. Ann. Intern. Med. 108:115-120.

22. Spiegelhalter, D. J. 1986. Probabilistic prediction in patient management and clinical trials. Stat. Med. 5:421-433.

23. Spiegelhalter, D. J., Abrams, K. R., and Myles, J. P. 2004. Bayesian Approaches to Clinical Trials and Health-Care Evaluation. John Wiley \& Sons, Ltd., Chichester, UK.

24. Spiegelhalter, D. J., and Knill-Jones R. P. 1984. Statistical and knowledge-based approaches to clinical decision support systems, with an application in gastroenterology (with discussion). J. R. Stat. Soc. A 147:35-77.

25. Teng, P. S., and Gaunt, R. E. 1980. Modelling systems of disease and yield loss in cereals. Agric. Syst. 6:131-154.

26. Tribus, M. 1969. Rational Descriptions, Designs and Decisions. Pergamon Press, New York.

27. Twengström, E., Sigvald, R., Svensson, C., and Yuen, J. 1998. Forecasting Sclerotinia stem rot in spring sown oilseed rape. Crop Prot. 17:405-411.

28. Van den Ende, J., Bisoffi, Z., Van Puymbroek, H., Van Gompel, A., Derese, A., Lynen, L., Moreira, J., and Janssen, P. A. J. 2007. Bridging the gap between clinical practice and diagnostic clinical epidemiology: Pilot experiences with a didactic model based on a logarithmic scale. J. Eval. Clin. Pract. 13:374-380.

29. Van den Ende, J., Moreira, J., Basinga, P., and Bisoffi, Z. 2005. The trouble with likelihood ratios. Lancet 366:548.

30. van der Plank, J. E. 1963. Plant Diseases: Epidemics and Control. Academic Press, New York.

31. Yuen, J. E., and Hughes, G. 2002. Bayesian analysis of plant disease prediction. Plant Pathol. 51:407-412.

32. Yuen, J., Twengström, E., and Sigvald, R. 1996. Calibration and verification of risk algorithms using logistic regression. Eur. J. Plant Pathol. 102:847-854. 